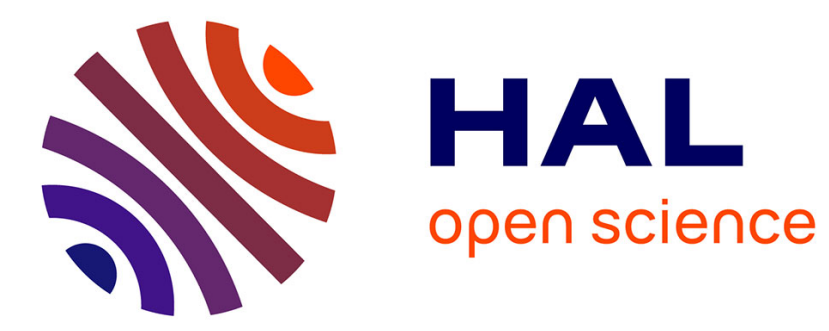

\title{
Sound-insulation layer modelling in car computational vibroacoustics in the medium-frequency range
}

C. Fernandez, Christian Soize, L. Gagliardini

\section{To cite this version:}

C. Fernandez, Christian Soize, L. Gagliardini. Sound-insulation layer modelling in car computational vibroacoustics in the medium-frequency range. Acta Acustica united with Acustica, 2010, 96 (3), pp.437-444. 10.3813/AAA.918296 . hal-00684316

\section{HAL Id: hal-00684316 \\ https://hal.science/hal-00684316}

Submitted on 1 Apr 2012

HAL is a multi-disciplinary open access archive for the deposit and dissemination of scientific research documents, whether they are published or not. The documents may come from teaching and research institutions in France or abroad, or from public or private research centers.
L'archive ouverte pluridisciplinaire HAL, est destinée au dépôt et à la diffusion de documents scientifiques de niveau recherche, publiés ou non, émanant des établissements d'enseignement et de recherche français ou étrangers, des laboratoires publics ou privés. 


\title{
Sound-insulation layer modelling in car computational vibroacoustics in the medium-frequency range
}

\author{
Charles Fernandez and Christian Soize*, \\ Universite Paris-Est, \\ Laboratoire Modelisation Simulation Multi-Echelle FRE3160 CNRS, \\ 5 bd Descartes, 77454 Marne-la-Vallee, France \\ Laurent Gagliardini, \\ PSA Peugeot-Citroen, CTV, route de Gisy, \\ 78943 Velizy-Villacoublay Cedex France
}

November 7, 2009

*corresponding author : christian.soize@univ-paris-est.fr 


\begin{abstract}
In a previous article, a simplified low- and medium-frequency model for uncertain automotive sound-insulation layers was developed and experimentally identified and validated. This model is based on a stochastic elastoacoustic element whose mean part comes from an extension of the fuzzy structures theory and depends on three physical parameters: the modal density, the damping rate and the participating mass. A non-parametric probabilistic approach is used to build the uncertainty-accounting stochastic simplified model. This model takes into account the modelling and system-parameters uncertainties and depends on three dispersion parameters. In this paper, the insulation simplified model is implemented in an industrial stochastic vibroacoustic model of a car. An experimental database of tests on vehicles has been carried out and is compared with the predictions. The analysis of these results shows the relevance of the proposed methodology for complex vibroacoustics industrial computational models.
\end{abstract}

Keywords: Theory of fuzzy structures, computational structural-acoustics, complex structures, uncertainties, non-parametric probabilistic approach, tests. 


\section{Introduction}

This paper is devoted to the application of a recently developed robust model of soundinsulation layers for computational vibroacoustics simulation of complex systems in lowand medium-frequency ranges. The sound-insulation layers will be designated here as insulations. In general, the insulations used by the car manufacturer are modelled by an added mass although they behave as a resonant continuous dynamical system in the frequency band of interest. Therefore, the internal resonances of the insulations are not taken into account contrary to the model used in this paper. All the theory used for the industrial application and summarized below has been developed and experimentally validated in references $[1,2]$. The insulation simplified model has been built in extending the fuzzy structures theory to a stochastic elastoacoustic element. This theory was introduced in $[3,4,5]$ and its original concept was reused in $[6,7,8,9,10,11]$. The mean part of the insulation stochastic model has been constructed with the fuzzy structures theory and the fluctuations around the mean have been built using the non-parametric probabilistic approach (see $[12,13])$. This model takes into account both model uncertainties and system-parameters uncertainties. The sound-insulation layer is assumed to behave as a resonant continuous dynamical system in the frequency band of interest. In this paper, we will not consider the high-frequency range where more elastic modes may appear. For such a modeling in the low- and medium-frequency ranges, an approach consists in modeling a sound-insulation layer as a poro-elastic medium using the Biot theory; the finite element method is then classically used to solve the associated boundary value problem. In this case, both vibroacoustic system and sound-insulation layers are modeled by the finite element method (see for instance [14, 15, 16, 17, 18, 19, 20, 21]). When the first thickness eigenfrequencies belong to the frequency band of analysis such a finite element model of sound-insulation layer introduces a large number of physical degrees of freedom (DOF) in the computational model as well as numerous elastic modes in the band. The size of the associated reduced computational model is then increased a lot. For instance, in a car booming noise analysis (frequency range [100, 250] Hz later referred as low frequency range), the finite element model may involve up to two millions of DOF for the structure and the reduced model requires about one thousand elastic modes. If the sound-insulation layers were modeled by the finite element method, an additional number of about five millions of DOF would be necessary. Twenty thousand additional elastic modes then appear in the reduced computational model exceeding the limits of current computational ressources. Consequently, there is a great interest to construct simplified sound-insulation layer models without adding neither physical DOF nor generalized DOF. Representing the sound-insulation layer by an adapted wall impedance can be a way to avoid the increase of DOF number (see for instance [22, 23, 24, 25, 26, 27, 28, 29, 30, 31] for the wall-acoustic impedances).

In order to lighten the understanding, a summary of the stochastic model construction is presented below.

(1) As explained above, a complete three dimensional modelling of the insulation would consist in introducing a poroelastic medium and such an approach would introduce a large additional number of DOF in the computational model. The proposed mean simplified 
model is constructed using the fuzzy structure theory which captures the main dynamical physical phenomena into insulations without introducing additional degrees of freedom and which is described in terms of fundamental physical parameters. In the frequency band of interest, the major phenomena are due to the internal resonances of the insulation. The fuzzy structure theory allows hidden dynamical degrees-of-freedom effects on the master structure to be taken into account in the sense of statistical averaging. With such a theory, the power flow between an fuzzy subsystem (an insulation) and the master vibroacoustic system (the car) is mainly controlled by the number of resonances in the insulation, by the participating dynamical mass and by the internal damping. The corresponding parameters of the mean model of an insulation are then its mean modal density of internal resonances, its mean coefficient of participating mass defined with respect to its total physical mass and finally, its mean damping rate.

(2) The mean parameters of an insulation simplified model, that is to say, the modal density, the coefficient of participating mass and the damping rate are identified following the methodology proposed in $[1,2]$ which is briefly summarized below to facilitate the reading.

(3) The model and system-parameters uncertainties are taken into account with the nonparametric probabilistic approach. This will not be detailed in this paper and one refers the reader to $[32,33,1,2]$.

(4) The insulation probabilistic simplified model depends on three dispersion parameters whose identification methodology is not described here. One refers again the reader to $[34,1,2]$.

An extensive database of experimental Frequency Response Functions (FRF) has been used to validate the computational prediction.

In Section 2, one summarizes the complete stochastic computational vibroacoustic model including insulations. Section 3 is devoted to the presentation of a new approach for the identification of the insulation mean model parameters. Section 4 deals with the application to the car booming noise analysis and an experimental validation is presented.

\section{Insulation modelling for computational vibroacous- tic model}

\subsection{Mean reduced computational vibroacoustic model without insulation}

The physical space $\mathbb{R}^{3}$ is referred to a cartesian system for which the generic point is denoted by $\boldsymbol{x}=\left(x_{1}, x_{2}, x_{3}\right)$. The Fourier transform with respect to time $t$ is denoted by $u(\omega)=\int_{\mathbb{R}} e^{-i \omega t} u(t) d t$. The vibroacoustic system is analyzed in the frequency band $\mathbb{B}=\left[\omega_{\min }, \omega_{\max }\right]$. For all $\omega$ in $\mathbb{B}$, the mean computational model of the car structure coupled with the acoustic cavity without the insulation simplified model (see Figure 1) is 


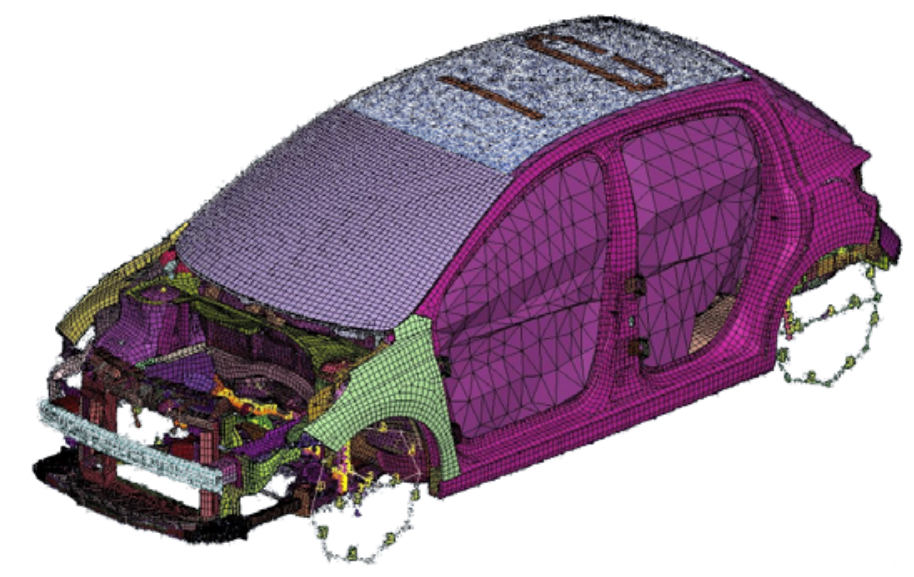

Figure 1: Computational vibroacoustic model of the car structure coupled with the acoustic cavity

written as

$$
\left[\begin{array}{cc}
{\left[\mathbb{A}^{s}(\omega)\right]} & {[\mathbb{C}]} \\
\omega^{2}[\mathbb{C}]^{T} & {\left[\mathbb{A}^{a}\right](\omega)}
\end{array}\right]\left[\begin{array}{c}
\boldsymbol{u}^{s}(\omega) \\
\boldsymbol{p}(\omega)
\end{array}\right]=\left[\begin{array}{c}
\mathbb{E}^{s}(\omega) \\
\mathbb{\mathbb { F }}^{a}(\omega)
\end{array}\right],
$$

where

$$
\begin{gathered}
{\left[\mathbb{A}^{s}(\omega)\right]=-\omega^{2}\left[\mathbb{M}^{s}\right]+i \omega\left[\mathbb{D}^{s}(\omega)\right]+\left[\mathbb{K}^{s}(\omega)\right]} \\
{\left[\mathbb{A}^{a}(\omega)\right]=-\omega^{2}\left[\mathbb{M}^{a}\right]+i \omega\left[\mathbb{D}^{a}(\omega)\right]+\left[\mathbb{K}^{a}\right] .}
\end{gathered}
$$

The mean matrices $\left[\mathbb{M}^{s}\right]$ (structural mass matrix), $\left[\mathbb{D}^{s}(\omega)\right]$ (structural damping matrix), $\left[\mathbb{K}^{s}(\omega)\right]$ (structural stiffness matrix), $\left[\mathbb{M}^{a}\right]$ (acoustic "mass" matrix), $\left[\mathbb{D}^{a}(\omega)\right]$ (acoustic damping matrix), $\left[\mathbb{K}^{a}\right]$ (acoustic "stiffness" matrix) and $[\mathbb{C}]$ (structural-acoustics coupling matrix on the interface without insulation) are constructed with the finite element method. The mean reduced system is obtained by projection on the elastic modes of the structure in vacuo and the acoustic modes of the acoustic cavity with rigid walls which is written as

$$
\left[\begin{array}{cc}
{\left[A^{s}(\omega)\right]} & {[C]} \\
\omega^{2}[C]^{T} & {\left[A^{a}\right](\omega)}
\end{array}\right]\left[\begin{array}{l}
\boldsymbol{q}^{s}(\omega) \\
\boldsymbol{q}^{a}(\omega)
\end{array}\right]=\left[\begin{array}{l}
\boldsymbol{f}^{s}(\omega) \\
\boldsymbol{f}^{a}(\omega)
\end{array}\right],
$$

where $\boldsymbol{f}^{s}$ is the generalized forces applied to the structure, $\boldsymbol{f}^{a}$ is the acoustic excitation, $\boldsymbol{q}^{s}$ is the generalized displacement of the structure and $\boldsymbol{q}^{a}$ is the generalized pressure in the acoustic cavity.

\subsection{Stochastic reduced computational vibroacoustic model in- cluding the insulation probabilistic simplified model}

For all $\omega$ in $\mathbb{B}$, the stochastic reduced computational model of the car structure coupled with the acoustic cavity and including the insulation probabilistic simplified model is 
written as

$$
\left[\begin{array}{cc}
{\left[\mathbf{A}^{s}(\omega)\right]+a^{s}(\omega)\left[\mathbf{B}^{s}\right]} & {[\mathbf{C}]+a^{c}(\omega)\left[\mathbf{C}^{s}\right]} \\
\omega^{2}\left\{[\mathbf{C}]^{T}+a^{c}(\omega)\left[\mathbf{C}^{s}\right]^{T}\right\} & {\left[\mathbf{A}^{a}\right](\omega)+a^{a}(\omega)\left[\mathbf{B}^{a}\right]}
\end{array}\right]\left[\begin{array}{c}
\mathbf{Q}^{s}(\omega) \\
\mathbf{Q}^{a}(\omega)
\end{array}\right]=\left[\begin{array}{c}
\boldsymbol{f}^{s}(\omega) \\
\boldsymbol{f}^{a}(\omega)
\end{array}\right],
$$

with

$$
\begin{gathered}
{\left[\mathbf{A}^{s}(\omega)\right]=-\omega^{2}\left[\mathbf{M}^{s}\right]+i \omega\left[\mathbf{D}^{s}(\omega)\right]+\left[\mathbf{K}^{s}(\omega)\right],} \\
{\left[\mathbf{A}^{a}(\omega)\right]=-\omega^{2}\left[\mathbf{M}^{a}\right]+i \omega\left[\mathbf{D}^{a}(\omega)\right]+\left[\mathbf{K}^{a}\right] .}
\end{gathered}
$$

The random matrices $\left[\mathbf{M}^{s}\right]$ (generalized structural mass matrix), $\left[\mathbf{D}^{s}(\omega)\right]$ (generalized structural damping matrix), $\left[\mathbf{K}^{s}(\omega)\right]$ (generalized structural stiffness matrix), $\left[\mathbf{M}^{a}\right]$ (generalized acoustic "mass" matrix), $\left[\mathbf{D}^{a}(\omega)\right]$ (generalized acoustic damping matrix), $\left[\mathbf{K}^{a}\right]$ (generalized acoustic "stiffness" matrix) and $[\mathbf{C}]$ (generalized structural-acoustics coupling matrix on the interface without insulations) are constructed with the non-parametric probabilistic approach introduced in 2000 (see $[12,13]$ ). The construction of these matrices is detailed in $[13,33]$. The random matrices $a^{s}(\omega)\left[\mathbf{B}^{s}\right]$ (insulation "effect" on the structure), $a^{c}(\omega)\left[\mathbf{C}^{s}\right]$ (insulation "effect" on the coupling interface) and $a^{a}(\omega)\left[\mathbf{B}^{a}\right]$ (insulation "effect" on the acoustic cavity) constitute the insulation probabilistic simplified model. They are constructed using the non-parametric probabilistic approach and the details are given in $[1,2]$. The matrices $\left[\mathbf{B}^{s}\right],\left[\mathbf{C}^{s}\right]$ and $\left[\mathbf{B}^{a}\right]$ depend only on the geometry of the structure and of the acoustic cavity. The three associated complex parameters $a^{s}(\omega), a^{c}(\omega)$ and $a^{a}(\omega)$ are constructed with the fuzzy structure theory. They depend on the material properties and on the mean parameters of the insulation mean simplified model. This dependency has been omitted for the sake of clarity in the presentation. Those parameters are written as

$$
\begin{gathered}
a^{s}(\mu, \xi, n, \omega)=-\omega^{2} a_{R}^{s}(\mu, \xi, n, \omega)+i \omega a_{I}^{s}(\mu, \xi, n, \omega), \\
a^{a}(\mu, \xi, n, \omega)=a_{R}^{a}(\mu, \xi, n, \omega)+i \omega a_{I}^{a}(\mu, \xi, n, \omega), \\
a^{c}(\mu, \xi, n, \omega)=a_{R}^{c}(\mu, \xi, n, \omega)+i a_{I}^{c}(\mu, \xi, n, \omega),
\end{gathered}
$$

where $\mu(\omega), \xi(\omega)$ and $n(\omega)$ are the mean participating mass, the mean damping rate and the mean modal density of the insulation (see [1,2] for the details).

\section{Identification of the mean parameters of the insu- lation mean simplified model}

In this section, a method for the estimation of the mean parameters of an insulation is presented. A computational model of the insulation is built using a very refined finite element model of the insulation and using nominal values for the parameters of this finite element model. The insulation is assumed to be embedded on its surface corresponding to the coupling interface with the structure. The generalized eigenvalue problem is then solved. The elastic mode $\varphi^{\alpha}$ associated with the eigenvalue $\lambda_{\alpha}$ is such that $\left[\mathbb{K}^{\text {ins }}\right] \varphi^{\alpha}=\lambda_{\alpha}\left[\mathbb{M}^{\text {ins }}\right] \varphi^{\alpha}$ where $\left[\mathbb{K}^{\text {ins }}\right]$ and $\left[\mathbb{M}^{\text {ins }}\right]$ are the usual stiffness and mass matrices of the insulation finite element model. The mean damping rate is chosen as the nominal value. Concerning 
the estimation of the mean participating mass coefficient, one proposes the following new approach. Let $N_{\text {tot }}$ be the total number of DOF of the insulation finite element model. Let $\mathcal{D}$ be a subset of all these DOF made up of $N$ DOF among these $N_{\text {tot }}$ DOF with $N \leq N_{\text {tot }}$ and such that

$$
\mathcal{D}=\left\{k_{1}, \ldots, k_{N}\right\} \quad .
$$

The kinetic energy associated with the mode $\alpha$ for all the DOF is such that

$$
\mathcal{E}_{N_{t o t}}^{\alpha}=\frac{1}{2}\left\{\boldsymbol{\varphi}^{\alpha}\right\}^{T}\left[\mathbb{M}^{\mathrm{ins}}\right] \boldsymbol{\varphi}^{\alpha} \dot{q}_{\alpha}^{2}
$$

where $\dot{q}_{\alpha}$ is the component $\alpha$ of the generalized velocity vector $\dot{\boldsymbol{q}}$. The kinetic energy associated with the mode $\alpha$ and with the $N$ DOF of the subset $\mathcal{D}$ is such that

$$
\mathcal{E}_{N}^{\alpha}=\frac{1}{2} \sum_{j=1}^{N} \sum_{\ell=1}^{N}\left[\mathbb{M}^{\mathrm{ins}}\right]_{k_{j} k_{\ell}} \varphi_{k_{j}}^{\alpha} \varphi_{k_{\ell}}^{\alpha} \dot{q}_{\alpha}^{2}
$$

Let $\mathcal{M}_{N}^{\alpha}$ be defined by

$$
\mathcal{M}_{N}^{\alpha}=\sum_{j=1}^{N} \sum_{\ell=1}^{N}\left[\mathbb{M}^{\mathrm{ins}}\right]_{k_{j} k_{\ell}} \varphi_{k_{j}}^{\alpha} \varphi_{k_{\ell}}^{\alpha}
$$

Using the normalization $\left\{\boldsymbol{\varphi}^{\alpha}\right\}^{T}\left[\mathbb{M}^{\text {ins }}\right] \varphi^{\alpha}=1$, it can be deduced that

$$
\mathcal{M}_{N_{\text {tot }}}^{\alpha}=1
$$

For the subset $\mathcal{D}$, the modal participating mass denoted by $m_{\alpha}$ is defined by

$$
\mathcal{E}_{N}^{\alpha}=\frac{1}{2} m_{\alpha} \frac{1}{N} \sum_{j=1}^{N}\left(\varphi_{k_{j}}^{\alpha}\right)^{2} \dot{q}_{\alpha}^{2}
$$

in which $\mathcal{E}_{N}^{\alpha}$ is defined by equation (13). The modal participating mass coefficient denoted by $\nu_{\alpha}$ is then defined by

$$
\nu_{\alpha}=\frac{m_{\alpha}}{m_{t o t}}
$$

where $m_{t o t}$ is the total mass of the insulation. Using equations (13), (14) and (17), it can be deduced that the mean modal participating mass coefficient can be written, for the subset $\mathcal{D}$, as

$$
\nu_{\alpha}=\frac{\mathcal{M}_{N}^{\alpha}}{\frac{m_{t o t}}{N} \sum_{j=1}^{N}\left(\varphi_{k_{j}}^{\alpha}\right)^{2}} .
$$

A selecting mode criterium to define the modes which contribute to the kinetic energy of the subset $\mathcal{D}$ is defined as follows:

$$
\text { the modes } \varphi^{\alpha} \text { such that } \frac{1}{N} \sum_{j=1}^{N}\left(\varphi_{k_{j}}^{\alpha}\right)^{2}<\frac{\mathcal{M}_{N}^{\alpha}}{m_{t o t}} \quad \text { are removed . }
$$



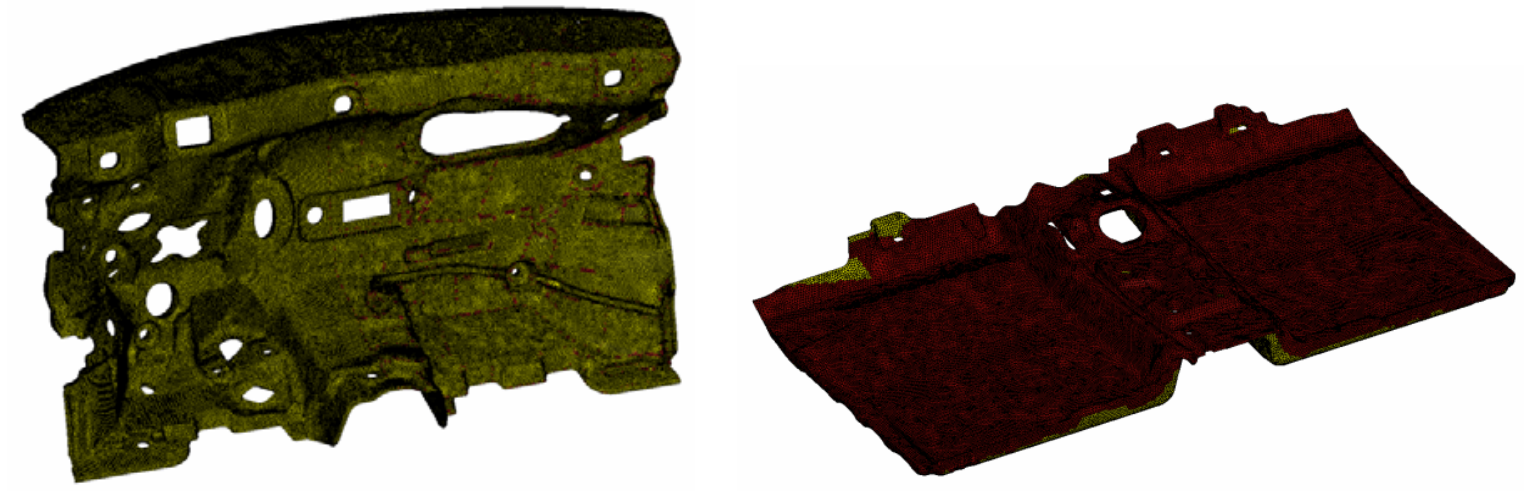

Figure 2: Finite element model of a dash insulation (left figure) and of a floor insulation (right figure) for a Peugeot 207 car.

The above inequality means that one removes the mode $\varphi^{\alpha}$ if $\nu_{\alpha}>1$. Since $\mathcal{M}_{N}^{\alpha} \leq$ $\left\{\boldsymbol{\varphi}^{\alpha}\right\}^{T}\left[\mathbb{M}^{\text {ins }}\right] \boldsymbol{\varphi}^{\alpha}=1$, it can be deduced that the criterium is such that

$$
\frac{1}{N} \sum_{j=1}^{N}\left(\varphi_{k_{j}}^{\alpha}\right)^{2}<\frac{\mathcal{M}_{N}^{\alpha}}{m_{t o t}} \leq \frac{1}{m_{t o t}}
$$

The use of the upper band value $1 / m_{\text {tot }}$ yields an error which is perfectly acceptable regarding the uncertainty in the insulation model. As an illustration on the selecting criterium used, for an insulation made up of a light foam covered with a heavy polymer layer, the subset $\mathcal{D}$ will be constituted of the DOF relative to the polymer layer. Finally, the mean modal density $n(\omega)$ is computed by calculating the number $\mathcal{N}(\omega)$ of eigenfrequencies in the band $[0, \omega]$ which yields

$$
\mathcal{N}(\omega)=\int_{0}^{\omega} n(\widetilde{\omega}) d \widetilde{\omega}
$$

\section{Application to the car vibroacoustic analysis and experimental validation}

In section 4.1, one presents the experimental measurements on a Peugeot 207 car for which the computational stochastic vibroacoustic model including insulations is used for the predictions. Section 4.2 is devoted to the identification of the mean parameters of the insulation mean simplified model for the dash and for the floor (see figure 2). Finally, an experimental validation and associated comments are presented in section 4.3. 

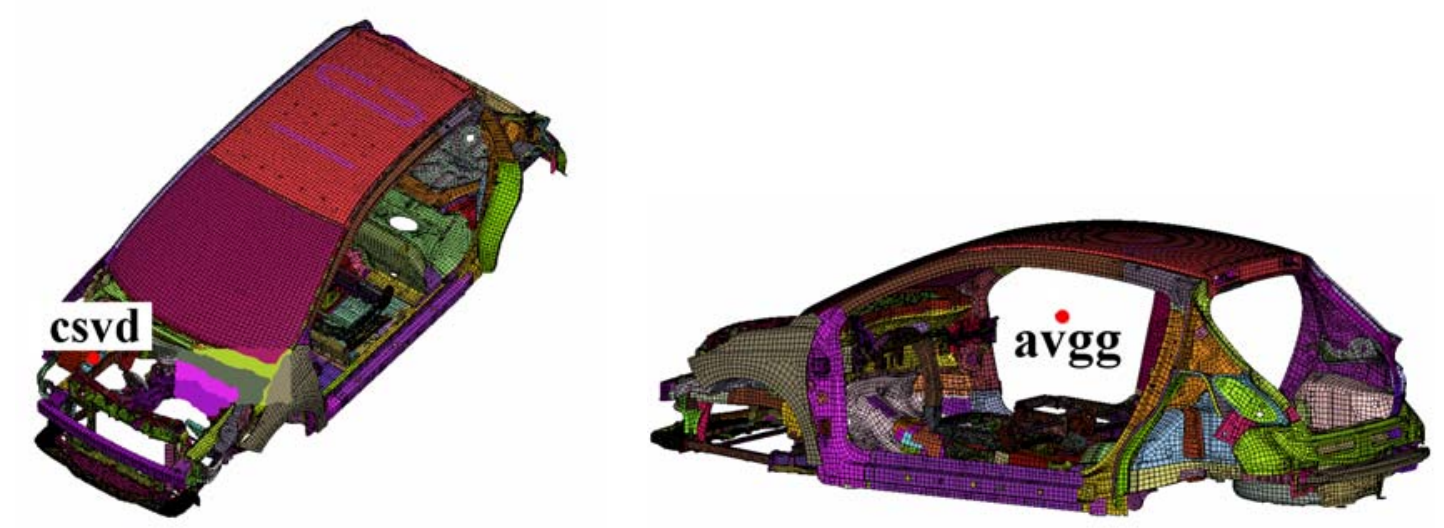

Figure 3: Experimental structural excitation (csvd) in vertical direction (left figure). Experimental acoustic observation (avgg) in pressure (right figure)

\subsection{Experimental measurements and stochastic computational vibroacoustic models}

The test protocol is similar to the protocol defined in [32]. The experimental database which is used below is made up of 40 vehicles of the same type (Peugeot 207 with 5 doors) measured at the end of the manufacturing process. These tests show a significant dispersion in the acoustic and vibration responses due to the manufacturing process and to optional extra. The complete database was made up of several excitation points and several observation points in different parts of the cars. In this paper, only a structuralacoustic response (measured 40 times) and a structural-vibratory response (measured 40 times) are presented. The vibratory excitation (force) point is located at the engine support in vertical direction (figures 3 and 4). The acoustic observation (pressure) point is located at the driver ears (figure 3). The vibratory observation (acceleration) point is located on the floor insulation in vertical direction (figure 4). For structural-vibratory FRF measurements, the excitation is performed by means of a hammer and the responses are identified with accelerometers. For the structural-acoustic FRF measurements, the reciprocity method in acoustics is used. This means that the excitation is produced by an acoustic source located at the driver ears inside the internal acoustic cavity and the acceleration responses are measured at the DOFs introduced above.

The mean computational vibroacoustic model is presented in figures 1 and 5 and has $1,175,909$ DOF. In order to show the improvement of the fuzzy-based insulation model, two reduced computational vibroacoustic models have been constructed and the predictions are then compared to the experiments. The first reduced computational model is made up of the finite element model of the structure coupled to the finite element model of the acoustic cavity and the effects of the insulations are taken into account by additional pure masses attached to the structure. This reduced computational model is constructed with 1,755 structural modes and 136 acoustic modes yielding a good convergence in the frequency band $[100,250] \mathrm{Hz}$. The second reduced computational model is made up of 

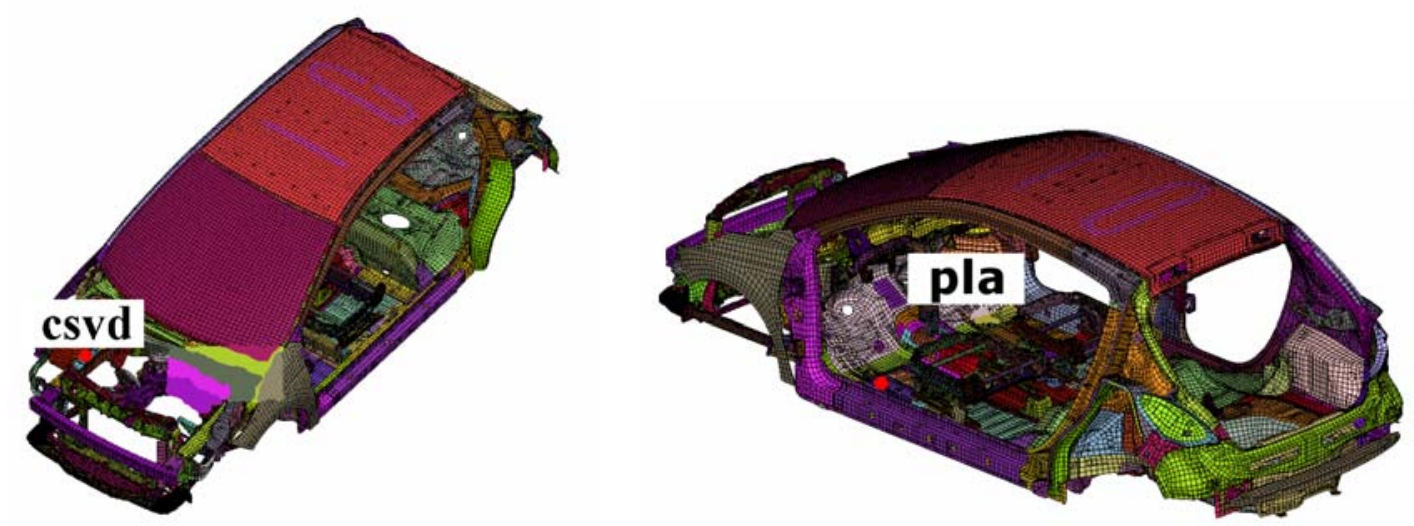

Figure 4: Experimental structural excitation (csvd) in vertical direction (left figure). Experimental vibratory observation (pla) in vertical direction (right figure)

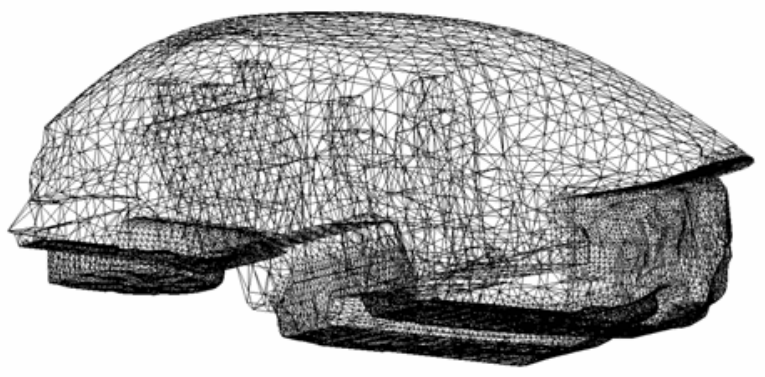

Figure 5: Computational model of the acoustic cavity 

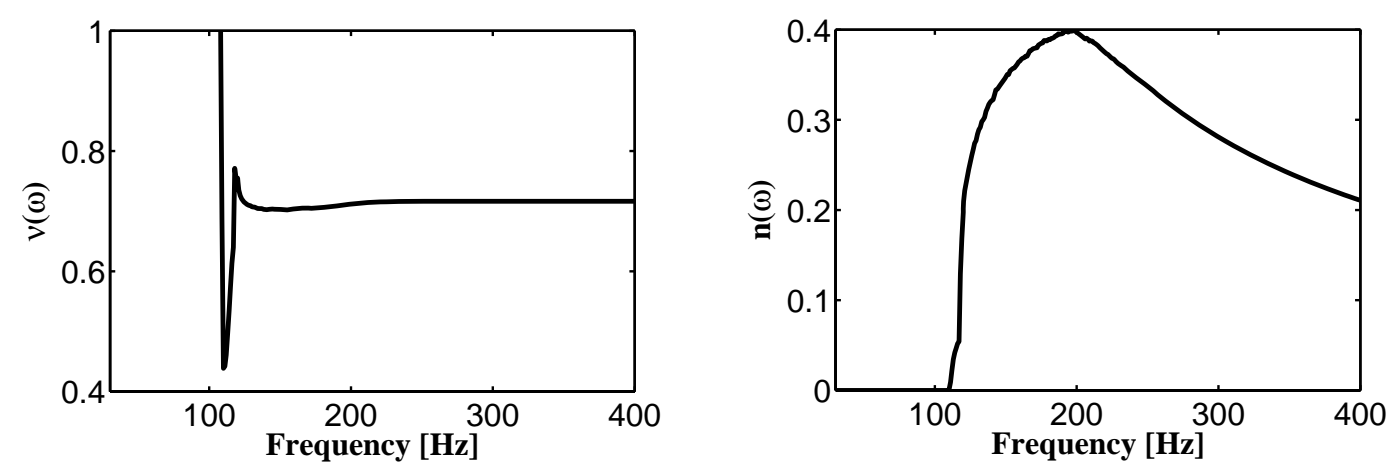

Figure 6: Mean participating mass coefficient (left) and mean modal density (right) for the dash insulation model

the stochastic vibroacoustic model including the insulation probabilistic simplified model and defined by equation (5). This stochastic reduced model is constructed with 1,700 structural modes and 136 acoustic modes yielding a good mean-square convergence in the frequency band $[100,250] \mathrm{Hz}$.

\subsection{Numerical identification of the parameters of the insulation mean simplified models}

The dash and floor insulations are made up of a light foam (poroelastic material) covered with a heavy polymer layer. The methodology described in section 3 is used to estimate the mean participating mass coefficient and the mean modal density from the refined finite element models displayed at figure 2 . Subset $\mathcal{D}$ defined in section 3 is constituted by the DOF of the heavy polymer layer in the normal direction. The eigenfrequencies and the modes for the DOF defined in the subset $\mathcal{D}$ are computed using the finite element model presented in figure 2 (with a geometry simplification) in order to apply the selecting modes criterium defined by equation (19). The insulation models are made up of flat shells, reproduce the geometry (dimensions, surfaces and thicknesses) and allow the internal dynamics of the structures to be captured. The methodology described in section 3 is then applied with a very low numerical cost. The finite element mesh used has 338,013 DOF for both the dash and floor insulations. The dash (resp. the floor) model surface is $1.38 \mathrm{~m}^{2}$ (resp. $1.02 \mathrm{~m}^{2}$ ) and its mean thickness is $0.025 \mathrm{~m}$ (resp. $0.025 \mathrm{~m}$ ). Figures 6 to 9 display the results obtained. The mean participating mass coefficient and the mean modal density for the dash (resp. the floor) are displayed in figure 6 (resp. in figure 8). The products $n(\omega) \nu(\omega)$ and the quotients $n(\omega) / \nu(\omega)$ for the dash (resp. the floor) are displayed in figure 7 (resp. in figure 9 ). These figures give the numerical values which are used in Section 4.3 for the stochastic computational vibroacoustic model concerning the mean parameters of the dash and floor insulation mean simplified models. It can be seen that the values of these mean parameters become significant above a frequency around 120 $\mathrm{Hz}$ which corresponds to the first thickness eigenfrequency of both insulations (dash and floor) and from this frequency, the insulations begin to act as a power flow transmitter. 

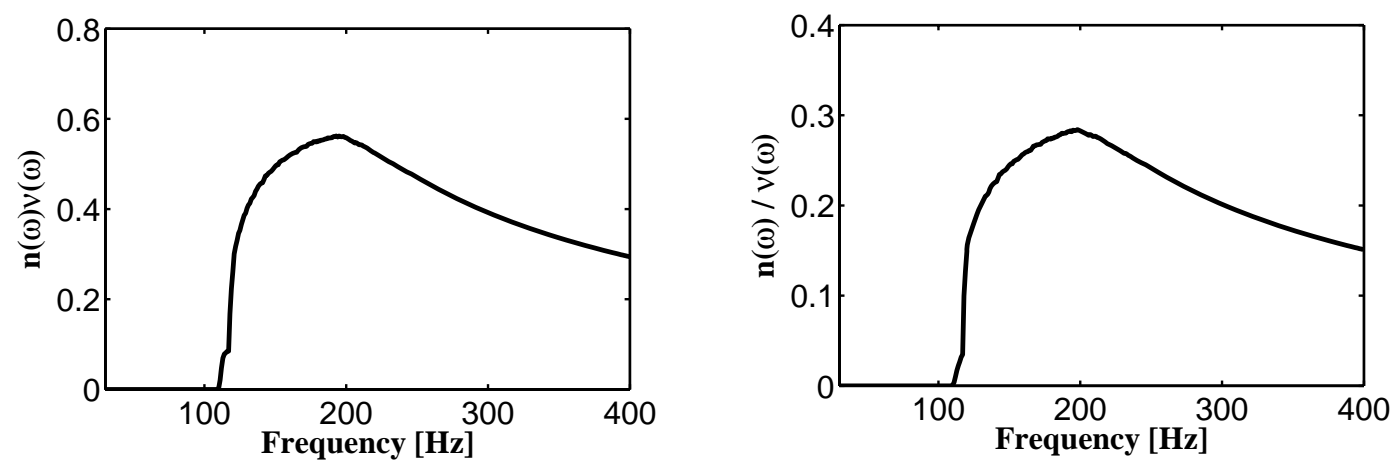

Figure 7: Product $n(\omega) \nu(\omega)$ (left) and quotient $n(\omega) / \nu(\omega)$ (right) for the dash insulation model
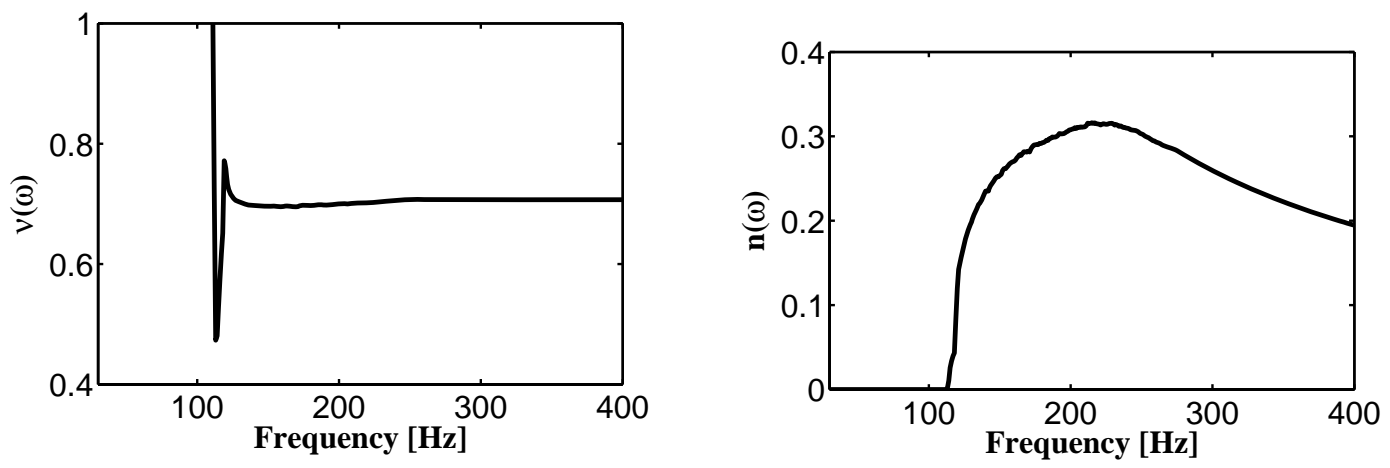

Figure 8: Mean participating mass coefficient (left) and mean modal density (right) for the floor insulation model
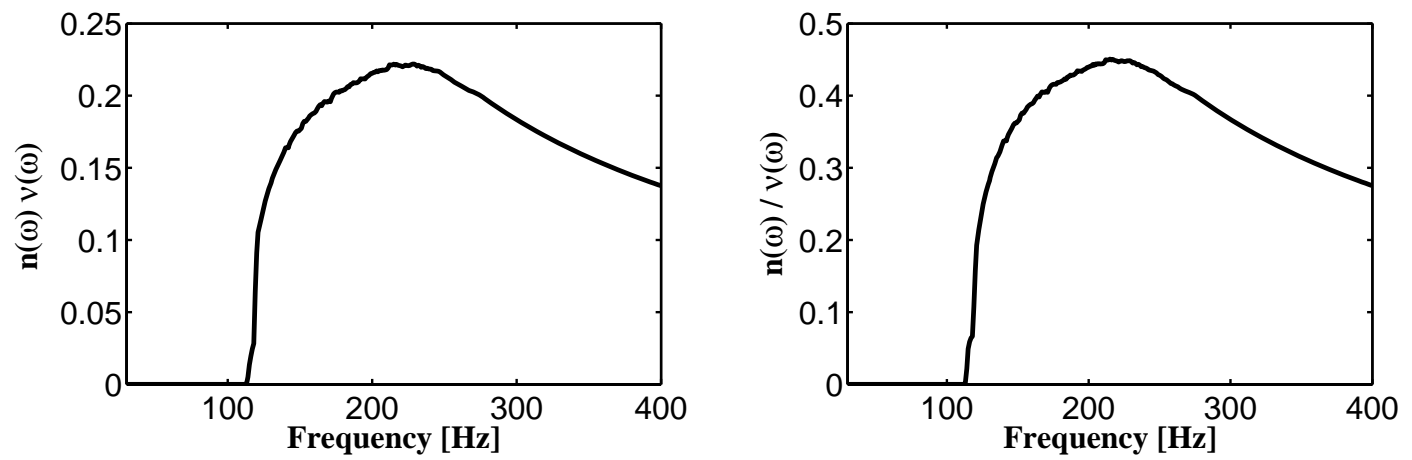

Figure 9: Product $n(\omega) \nu(\omega)$ (left) and quotient $n(\omega) / \nu(\omega)$ (right) for the floor insulation model 


\subsection{Experimental comparisons and analysis of the results}

The comparison between the measurements with the two stochastic computational models are presented in figure 10 (structural-acoustic responses) and in figure 11 (structuralvibration responses). In the four figures, the scales of the vertical axes are the same (10 $\mathrm{dB}$ between two consecutive ticks in the vertical axis) and the value "Ref" located at a tick corresponds to the same value in $\mathrm{dB}$ for the four figures. In figure 10 (resp. in figure 11), the level of the structural-acoustic response (resp. structural-vibration response) is displayed in $\mathrm{dB}$ as a function of the frequency for the band $[100,250] \mathrm{Hz}$. The 40 experimental FRF belong to the light grey domain (to the blue domain for the online version). The upper and lower thin lines are the maximum and minimum values for the experimental FRF and the middle line is the mean experimental response. The stochastic solution is computed by the Monte Carlo simulation with 1,000 realizations whose convergence is reached with 600 realizations. The level of uncertainty in the stochastic model is the one used and identified in $[33,1]$. The middle thick grey line represents the mean value of the stochastic response. The confidence region is constructed using the quantile method (see [35]) for a probability model $P_{c}=0.95$ (dark thick lines) and for $P_{c}=0.99$ (dark thick dotted lines). In both figures 10 and 11, the top figure is related to the stochastic computational vibroacoustic model for which the insulations are modelled by added masses. The down figure is related to the stochastic computational vibroacoustic model including the fuzzy-based insulation model.

(1) Firstly, the structural-acoustics response (figure 10) of the stochastic computational model including the insulation probabilistic simplified model shows an improvement of the prediction in frequency band $[120,160] \mathrm{Hz}$ for which the internal dynamics of the insulation plays a role. The first thickness eigenfrequency of both insulations (dash and floor) is located around $120 \mathrm{~Hz}$ and from this frequency, the insulations begin to act as a power flow transmitter. This internal dynamics is not predicted by the model with added masses. Secondly, the structural-vibration response (figure 11) of the stochastic computational model including the insulation probabilistic simplified model shows an improvement of the prediction in frequency band $[110,250] \mathrm{Hz}$. The observation point for the structural-vibration response is located on the insulation floor and the effect of the dissipation due to the internal dynamics is very strong at this place. The fuzzybased insulation modelling (the insulation probabilistic simplified model) improves the predictions in a robust framework with respect to both system-parameter uncertainties and model uncertainties.

(2) There remain errors which are directly due to modelling errors in the nominal model of the structure coupled with the acoustic cavity. However, these model uncertainties are taken into account with the non-parametric probabilistic model of uncertainties.

\section{Conclusion}

In this paper, an experimental validation of sound-insulation layers modelling applied to a real car vibroacoustics has been presented. The identification of the mean parameters 

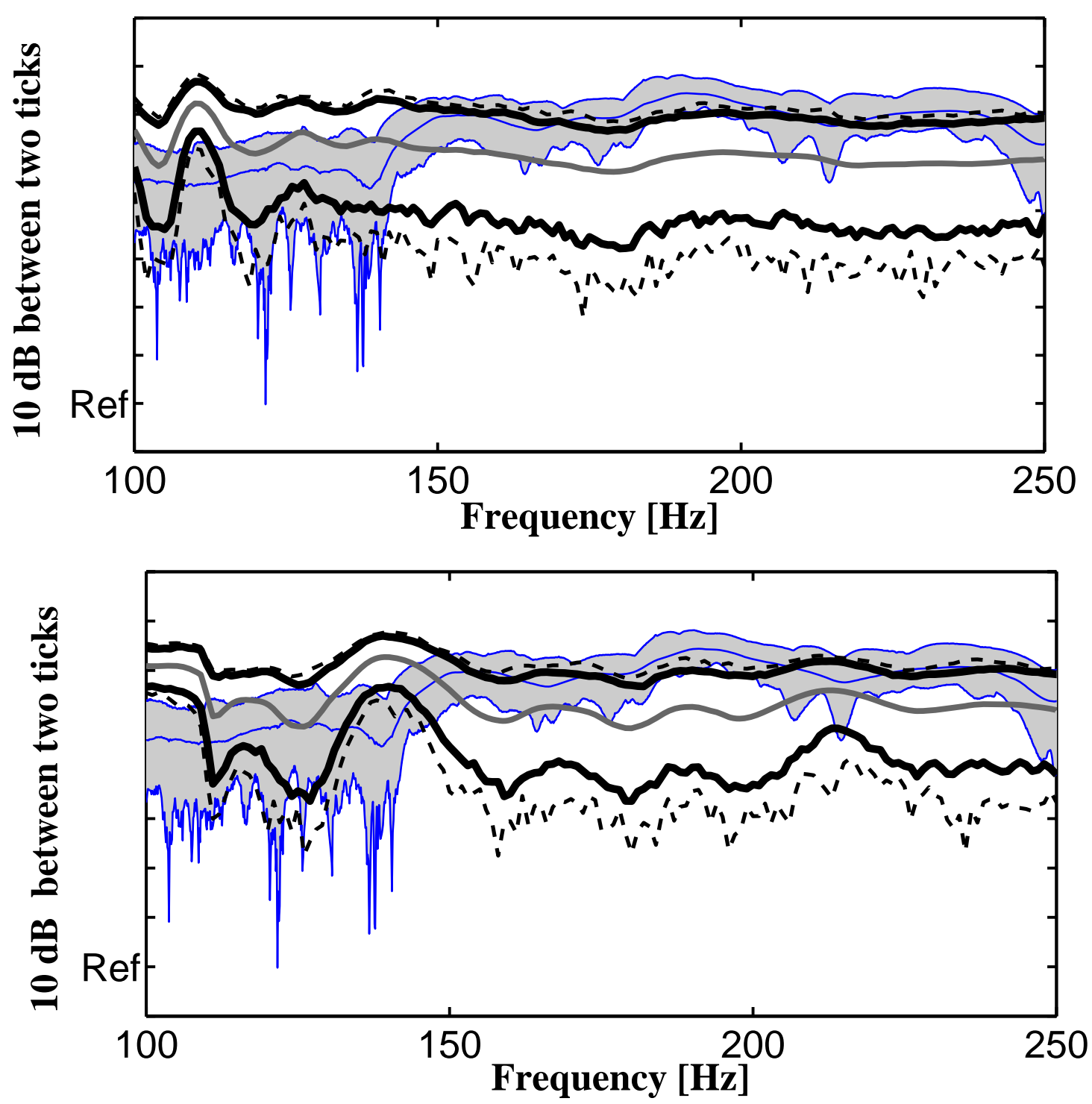

Figure 10: FRF in dB (vertical axis:10 dB between two consecutive ticks) for a mechanical excitation at point csvd and for an acoustic pressure observation at point avgg . Measurements (light grey domain or blue domain for the online version). Mean value of the stochastic response (thick grey line). Upper and lower envelopes of the confidence region of the stochastic response (dark thick lines for $P_{c}=0.95$ and dark thick dotted lines for $P_{c}=0.99$ ). Insulations effects modeled by added masses (top figure). Fuzzy-based insulation model (down figure). 

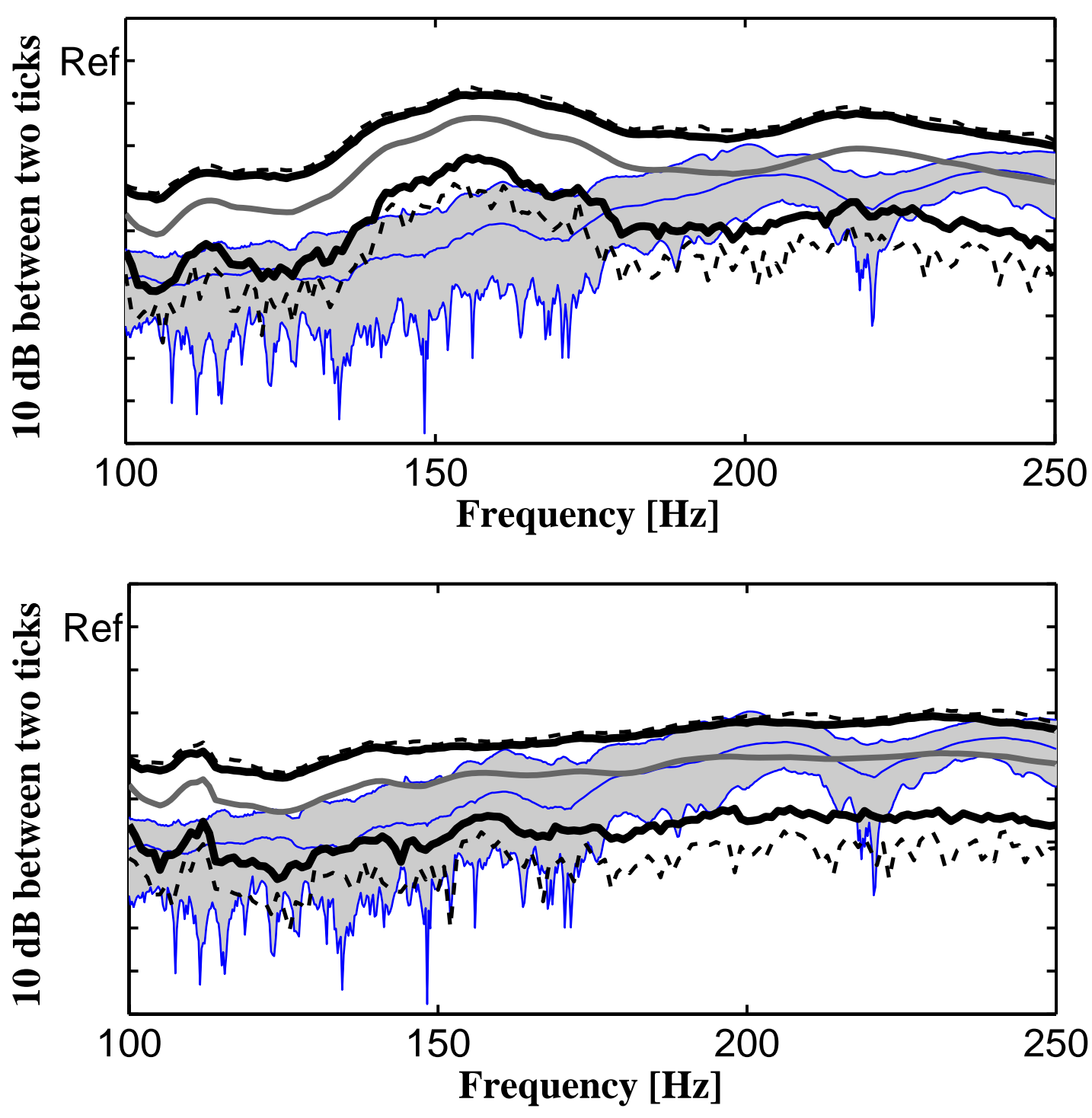

Figure 11: FRF in dB (vertical axis:10 dB between two consecutive ticks) for a mechanical excitation at point csvd and for an acceleration observation at point pla in vertical direction. Measurements (light grey domain or blue domain for the online version). Mean value of the stochastic response (thick grey line). Upper and lower envelopes of the confidence region of the stochastic response (dark thick lines for $P_{c}=0.95$ and dark thick dotted lines for $P_{c}=0.99$ ). Insulations effects modeled by added masses (top figure). Fuzzy-based insulation model (down figure). 
of the insulation mean simplified model, which are the mean modal density, the mean participating mass coefficient and the mean damping rate has been carried out with a novel approach for industrial sound-insulation layers (dash and floor). Although the nominal model (structure, acoustic cavity and vibroacoustic coupling) still contains errors (mainly due to the mean computational model of the nominal structure and of the acoustic cavity without insulations), the stochastic computational vibroacoustic model including the insulation probabilistic simplified model shows an improvement in the predictions compared to the usual modelling used by the manufacturer (added masses method).

In this case for which the first thickness eigenfrequency belongs to the frequency band of analysis, if a finite element model of the insulations were introduced then a large number of DOF would be added in the computational model. Moreover, the dimension of the associated reduced computational model would considerably be increased due to the presence of numerous elastic modes in the frequency band as it has been explained. In addition, the proposed model is more robust as it can be seen in the results which show that the confidence region is narrower in the frequency band containing the first thickness eigenfrequency than the computational models presently used by the manufacturers for which the insulation layers are modeled by added masses.

The stochastic computational predictions have been compared to an extensive database made up of same measures on 40 vehicles of the same type. This comparison validates the model, its parameters identification and the relevance of the proposed methodology. The fuzzy-based insulation modelling improves the predictions.

Acknowledgements This research has been supported by the French National Agency for Research and Technology (ANRT).

\section{References}

[1] C. Fernandez, C. Soize and L. Gagliardini, "Fuzzy structure theory modeling of insulations in complex vibroacoustic uncertain sytems - Theory and experimental validation", J. Acoust. Soc. Am., 125, 138-153 (2009).

[2] C. Fernandez, "Modelling and experimental validation of complex insulations for computational low- and medium-frequency vibroacoustics of cars", Doctoral thesis (in French), Université Paris-Est, France (2008).

[3] C. Soize, "A model and numerical method in the medium frequency range for vibroacoustic predictions using the theory of structural fuzzy", J. Acoust. Soc. Am., 94, 849-865 (1993). 
[4] C. Soize, "Estimation of the fuzzy substructure model parameters using the mean power flow equation of the fuzzy structure", Journal of Vibration and Acoustics, 120, 279-286 (1998).

[5] C. Soize and K. Bjaoui, "Estimation of fuzzy structure parameters for continuous junctions", Journal of Vibration and Acoustic, J. Sound Vib., 107, 2011-2020 (2000).

[6] A. D. Pierce, V. W. Sparrow and D. A. Russell, "Fundamental Structural-Acoustic Idealizations for Structures with Fuzzy Internals", Journal of Vibration and Acoustics, 117, 339-348 (1995).

[7] M. Strasberg and D. Feit, "Vibration damping of large structures induced by attached small resonant structures", J. Acoust. Soc. Am., 99, 335-344 (1996).

[8] G. Maidanik and J. Dickey, "Design criteria for the damping effectiveness of structural fuzzies", J. Acoust. Soc. Am., 100, 2029-2033 (1996).

[9] R. L. Weaver, "The effect of an undamped finite degree of freedom "fuzzy" substructure: Numerical Solution and theorical discussion", J. Acoust. Soc. Am., 100, 3159-3164 (1996).

[10] R. L. Weaver, "Mean and mean-square responses of a prototypical master/fuzzy structure", J. Acoust. Soc. Am., 101, 1441-1449 (1997).

[11] J.-M. Mencik and A. Berry, "A theoretical formulation of the dynamical response of a master structure coupled with elastic continuous fuzzy subsystems with discrete attachments ", J. Sound Vib., 280, 1031-1050 (2005).

[12] C. Soize, "Maximum entropy approach for modeling random uncertainties in transient elastodynamics", J. Acoust. Soc. Am., 109, 1979-1996 (2001).

[13] C. Soize, "Random matrix theory for modeling uncertainties in computational mechanics", Comput. Methods Appl. Mech. Engrg., 194, 1333-1366 (2005).

[14] Y.-J. Kang and J.S. Bolton, "Finite element modeling of isotropic elastic porous materials coupled with acoustical finite elements", J. Acoust. Soc. Am., 98, 635-643 (1995).

[15] Y.-J. Kang and J.S. Bolton, "A finite element model for sound transmission through foam-lined double-panel structures", J. Acoust. Soc. Am., 99, 2755-2765, (1996).

[16] N. Atalla and R. Panneton, "The effects of multilayer sound-absorbing treatments on the noise field inside a plate backed-cavity", J. Noise Control Eng., 44, 235-243 (1996).

[17] R. Panneton and N. Atalla, "Numerical prediction of sound transmission through finite multilayer systems with poroelastic materials", J. Acoust. Soc. Am., 100, 346354 (1996). 
[18] R. Panneton and N. Atalla, "An efficient finite element scheme for solving the threedimensional poroelasticity problem in acoustics", J. Acoust. Soc. Am., 101, 32873298 (1997).

[19] N. Atalla, M. A. Hamdi and R. Panneton, "Enhanced weak integral formulation for the mixed (u,p) poroelastic equations", J. Acoust. Soc. Am., 109, 3065-3068 (2001).

[20] L. Jaouen, B. Brouard, N. Atalla and C. Langlois, "A simplified numerical model for a plate backed by a thin foam layer in the low frequency range", J. Sound Vib., 280, 681-698 (2005).

[21] N. Atalla, F. Sgard and C. K. Amedin, "On the modeling of sound radiation from poroelastic materials", J. Acoust. Soc. Am., 120, 1990-1995 (2006).

[22] F. J. Fahy, Sound and Structural Vibration, Radiation, Transmission and Response (Academic, New York, 1985).

[23] A.D. Pierce, Acoustics: An Introduction to its Physical Principles and Applications (originally published in 1981, McGraw-Hill Acoust. Soc. Am., Publications on Acoustics, Woodbury, 1989).

[24] J.L. Guyader and C. Lesueur, "Acoustic transmission through orthotropic multilayered plates, part. I. Plate vibration modes", J. Sound Vib., 58, 51-86 (1978).

[25] J. A. Moore and R. H. Lyon, "Resonant porous material absorbers", J. Acoust. Soc. Am., 72, 1989-1999 (1982).

[26] J.F. Allard, C. Champoux and C. Depollier, "Modelization of layered sound absorbing materials with transfer matrices", J. Acoust. Soc. Am., 82, 1792-1796 (1987).

[27] M.C. Junger and D. Feit, Sound, Structures, and Their Interaction (originally published in 1972, Acoustical Society of America, New York, 1993).

[28] J.F Allard, Propagation of Sound in Porous Media: Modelling Sound Absorbing Materials (Chapman and Hall, London, 1994).

[29] B. Laulagnet, J.-L. Guyader, "Sound-radiation from finite cylindrical shells partially covered with longitudinal strips of compliant layers", J. Sound Vib., 186, 723-742 (1995).

[30] R. Ohayon and C. Soize, Structural Acoustics and Vibration (Academic press, San Diego, 1998).

[31] R. S. Langley and P. Bremner, "A hybrid method for the vibration analysis of complex structural-acoustic systems", J. Acoust. Soc. Am., 105, 1657-1671 (1999).

[32] J.-F. Durand, "Modélisation de Véhicules en Vibroacoustique Numérique avec Incertitudes de Modélisation et Validation Expérimentale", "Uncertain computational vibroacoustics modeling of vehicles including model uncertainties and experimental validation", Doctoral thesis, Université de Marne-la-Vallée, France (2007). 
[33] J.-F. Durand, C. Soize and L. Gagliardini, "Structural-acoustic modeling of automotive vehicles in presence of uncertainties and experimental identification and validation", J. Acoust. Soc. Am., 24, 1513-1525 (2008).

[34] C. Soize, E. Capiez-Lernout, J.-F. Durand, C. Fernandez and L. Gagliardini, "Probabilistic model identification of uncertainties in computational models for dynamical systems and experimental validation", Comput. Methods Appl. Mech. Engrg., 198, 150-163 (2008).

[35] R. Serfling, Approximations Theorems of Mathematical Statistics (John Wiley and Sons, New York, 1980). 